\title{
To consider the electromagnetic field as fundamental, and the metric only as a subsidiary field
}

\author{
Friedrich W. Hehl* \\ Institute for Theoretical Physics, \\ University of Cologne, \\ 50923 Köln, Germany \\ and \\ Department of Physics and Astronomy, \\ University of Missouri-Columbia, \\ Columbia, MO 65211, USA \\ Yuri N. Obukhovi \\ Institute for Theoretical Physics, \\ University of Cologne, \\ 50923 Köln, Germany \\ and \\ Dept. Theor. Physics, \\ Moscow State University, \\ 117234 Moscow, Russia
}

(Dated: 21 April 2004, file forerunner5.tex)

In accordance with an old suggestion of Asher Peres (1962), we consider the electromagnetic field as fundamental and the metric as a subsidiary field. In following up this thought, we formulate Maxwell's theory in a diffeomorphism invariant and metric-independent way. The electromagnetic field is then given in terms of the excitation $H=(\mathcal{H}, \mathcal{D})$ and the field strength $F=(E, B)$. Additionally, a local and linear "spacetime relation" is assumed between $H$ and $F$, namely $H \sim \kappa F$, with the constitutive tensor $\kappa$. The propagation is studied of electromagnetic wave fronts (surfaces of discontinuity) with a method of Hadamard. We find a generalized Fresnel equation that is quartic in the wave covector of the wave front. We discuss under which conditions the waves propagate along the light cone. Thereby we derive the 
metric of spacetime, up to a conformal factor, by purely electromagnetic methods.

PACS numbers: 03.50.De, 04.20.Cv

Keywords: Classical electrodynamics, premetric axiomatics, differential forms, electric/magnetic reciprocity, light cone, metric

\section{INTRODUCTION}

"It is therefore suggested to consider the electromagnetic field as fundamental, and the metric field only as a subsidiary quantity." This is a quotation from an article that Asher Peres 19] wrote in 1962. Asher's idea was to start with the source-free Maxwell equations

$$
d H=0, \quad d F=0
$$

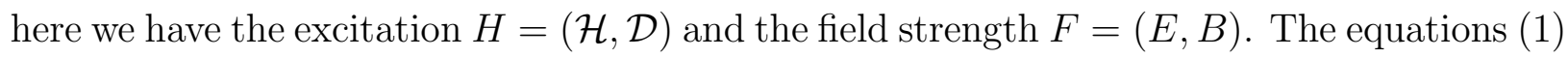
are diffeomorphism invariant and independent of the metric $g_{i j}(x)$ of spacetime. In other words, this is the premetric form [3, 11, 12, 22, 30, 31] of Maxwell's equations (without source). The excitation $H$ and the field strength $F$ are considered to be directly measurable quantities, via Maxwellian double place in the case of $\mathcal{D}$ and via the Coulomb force in the case of $E$; analogous procedures exist for $\mathcal{H}$ and $B$, respectively, see [4].

Peres then considered the "constitutive" law relating $H$ and $F$ in vacuum, namely

$$
H=\lambda^{\star} F,
$$

as a definition of the metric; $\lambda$ is a universal constant and $\star$ the (metric dependent) Hodge star operator. In components the metric is displayed more explicitly,

$$
\check{H}^{i j}=\frac{\lambda}{2} \sqrt{-g} g^{i k} g^{j \ell} F_{k \ell},
$$

with $\check{H}^{i j}=\frac{1}{2} \epsilon^{i j k \ell} H_{k \ell}$, where we used the totally antisymmetric Levi-Civita symbol $\epsilon^{i j k \ell}=$ $\pm 1,0$. The components of the excitation and field strength 2 -forms are given by $H=$ $H_{i j} d x^{i} \wedge d x^{j} / 2$ and $F=F_{i j} d x^{i} \wedge d x^{j} / 2$, respectively. The components $\check{H}^{i j}$ and $F_{k \ell}$ are

*Electronic address: hehl@thp.uni-koeln.de

†Electronic address: yo@thp.uni-koeln.de 
assumed to be known and (3) has to be resolved with respect to the metric $g_{i j}$. This was the program of Peres 19] for deriving the metric from the electromagnetic field.

Without doubt, Peres is the forerunner of the movement to construct the metric of spacetime out of purely electromagnetic data. The algebraic method used by Peres in his attempt was analyzed in detail by Rubilar [23]. Subsequent to Peres [19], Toupin [30] and Schönberg 27] proved the existence of a metric in this context. Nowadays we know [4] that the program, as layed out by Peres, can be explicitly implemented. In this paper, which we would like to dedicate to Asher Peres on the occasion 70th birthday, we are going to sketch this procedure.

Notation (see [4]): We use the formalism of exterior differential forms. We denote the frame by $e_{\alpha}$, with the anholonomic or frame indices $\alpha, \beta, \cdots=0,1,2,3$. Decomposed with respect to a natural frame $\partial_{i}$, we have $e_{\alpha}=e_{\alpha}^{i} \partial_{i}$, where $i, j, \cdots=0,1,2,3$ are holonomic or coordinate indices. The frame $e_{\alpha}$ is the vector basis of the tangent space at each point of the $4 \mathrm{D}$ spacetime manifold. The symbol $\rfloor$ denotes the interior and $\wedge$ the exterior product. Both products are metric independent. The coframe $\vartheta^{\beta}=e_{j}^{\beta} d x^{j}$ is dual to the frame, i.e., $\left.e_{\alpha}\right\rfloor \vartheta^{\beta}=\delta_{\alpha}^{\beta}$.

\section{PREMETRIC ELECTRODYNAMICS}

Since electric charges are occurring in nature in integer multiples of $e / 3$, here $e$ is the elementary charge, they can be counted. Accordingly, if we consider a 3-dimensional (3D) volume, we can count how many elementary charges are contained in it. Macroscopically in 4 dimensions (4D), we can describe the charge density and its flux by the 3 -form $J$ that is conserved:

$$
d J=0 .
$$

If the global version of charge conservation (4) is suitably formulated, by de Rham's theorem the electric current $J$ turns out to be exact:

$$
J=d H
$$

This is the inhomogeneous Maxwell equation, with the excitation 2-form $H$.

In deriving (4) and (5), only the ability to define an arbitrary 4D volume is necessary together with the counting of electric charges. No distance measurement nor any paral- 
lel transfer is involved. Therefore it is evident that the inhomogeneous Maxwell equation already exists on a 4D manifold without metric and without linear connection.

With the help of the Lorentz force density

$$
\left.f_{\alpha}=\left(e_{\alpha}\right\rfloor F\right) \wedge J
$$

we can define the electromagnetic field strength 2-form $F$. If $F$ is integrated over a 2D area, it describes the magnetic flux lines piercing through this area.

In certain situations, inside a superconducting region of a superconductor of type II, magnetic flux lines are quantized and can be counted. Here again, the counting of certain units, now the magnetic flux quanta, together with the ability to circumscribe a $2 \mathrm{D}$ area element, is all what is needed to formulate a conservation law. This conservation law of magnetic flux we assume to be generally valid:

$$
d F=0
$$

Again, no metric and no linear connection of spacetime is involved. As we will discuss below, Faraday's induction law is a consequence of (77) thereby giving (17) a firm experimental basis.

Also without using a metric, we can decompose the fields entering the Maxwell equations into 1 plus 3 dimensions [4],

$$
H=-\mathcal{H} \wedge d \sigma+\mathcal{D}, \quad J=-j \wedge d \sigma+\rho
$$

and

$$
F=E \wedge d \sigma+f_{\lambda} B, \quad A=-\varphi d \sigma+\mathcal{A}
$$

Here $\sigma$ is the prototype of a time variable. The Lenz factor we put to one in accordance with the Lenz rule, $f_{\lambda}=+1$, see [8] for details. We also decomposed the potential 1-form $A$ that is defined by $F=d A$.

Using these decompositions and substituting them into the Maxwell equations (5) and (17), we find the conventional form of the Maxwell equations,

$$
d H=J\left\{\begin{array}{rl}
\underline{d} \mathcal{D} & =\rho, \\
\dot{\mathcal{D}} & =\underline{d} \mathcal{H}-j,
\end{array} \quad d F=0 \quad\left\{\begin{aligned}
\underline{d} B & =0, \\
\dot{B} & =-\underline{d} E,
\end{aligned}\right.\right.
$$

cf. Sommerfeld [29] and Scheck [26]. The 3D exterior derivative is denoted by $\underline{d}$, the time derivative by a dot. 

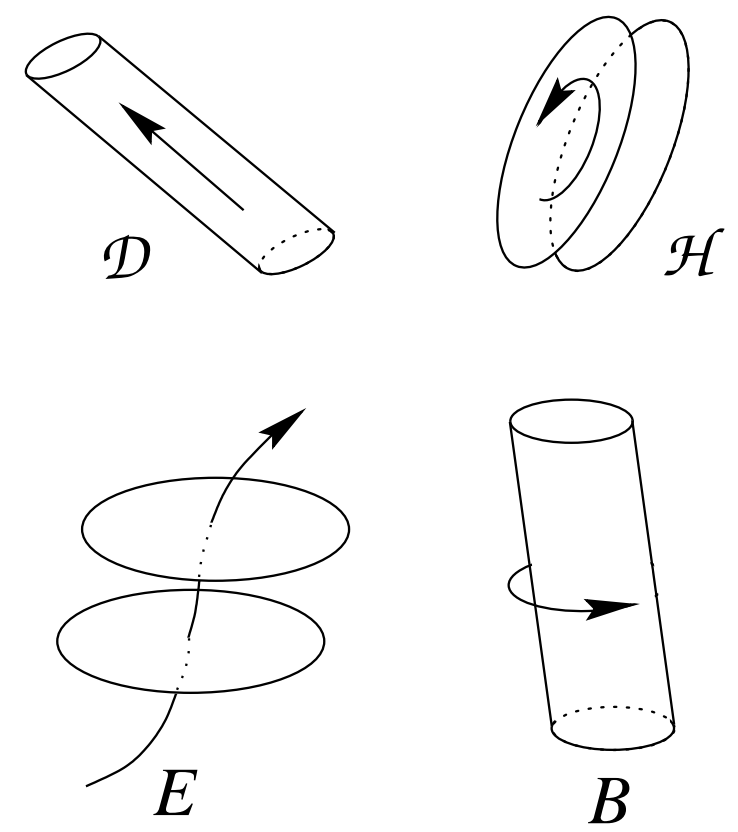

FIG. 1: Faraday-Schouten pictograms of the electromagnetic field in 3-dimensional space. The images of 1-forms are represented by two neighboring planes. The nearer the planes, the stronger the 1-form is. The 2-forms are pictured as flux tubes. The thinner the tubes are, the stronger the flow is. The difference between a twisted and an untwisted form accounts for the two different types of 1- and 2-forms, respectively. Taken from Hehl and Obukhov [4].

We can complete this premetric electrodynamics by substituting (5) into (6). Then, by some algebra and by some physical arguments, one ends up with an axiom for the energymomentum current 3-form of the electromagnetic field:

$$
\left.\left.\Sigma_{\alpha}:=\frac{1}{2}\left[F \wedge\left(e_{\alpha}\right\rfloor H\right)-H \wedge\left(e_{\alpha}\right\rfloor F\right)\right]
$$

In equations (44) to (11), we collected all fundamental relations of classical electrodynamics. They are all diffeomorphism invariant, that is, completely independent of the coordinates used. With the exceptions of (6) and (11), the equations are also invariant under arbitrary linear frame transformations. In contrast, eqs.(6) and (11), like the frame $e_{\alpha}$, transform linearly, that is, they are covariant under linear frame transformations.

We started by counting electric charges and magnetic flux lines, used no metric (i.e., no gravitational potential) and no linear connection of spacetime, and found a generally covariant basis for electrodynamics. In particular, there is no relation whatsoever to the Poincaré group (also known as inhomogeneous Lorentz group). It simply does not enter at 
that level of our setting up of classical electrodynamics. Classical electrodynamics is not so closely related to special relativity as most representations in textbooks want to make us believe.

The present framework can be suitably generalized in order to allow for magnetic charges (tantamount of violating magnetic flux conservation, i.e., $d F \neq 0$ ), see [5, 9]. However, since magnetic charges have never been found experimentally, we will not follow this train of thought any further.

In contrast, the violation of electric charge conservation, i.e., $d J \neq 0$, would probably hit our axiomatic set-up fatally. Firstly, the inhomogeneous Maxwell equation (5) would be lost and secondly, the Lorentz force density (6), with a non-conserved charge, is no longer expected to qualify as a bona fide defining equation for the electromagnetic field strength F. Lämmerzahl et al. [14, 15, 16] developed an electrodynamic theory in the framework of which one can accommodate a violation of electric charge conservation. They use it as a test theory for interpreting corresponding experiments. They propose such experiments in order to improve the experimental bounds for charge conservation.

Since in elementary particle physics all evidence supports electric charge conservation, we will stick to this principle and will continue our considerations by assuming the validity of (41) and (7), respectively.

One could ask why these two conservation laws are so "stable". The absolute dimensions of current and field strength are $[J]=q$ and $[F]=h / q$, respectively, with $q \sim$ dimension of charge and $h \sim$ dimension of action. Let us assume that the elementary charge $e$ and Planck's constant $\hbar$ are really constants of nature, that is, neither time nor space dependent. Then the elementary quanta of charge $e / 3$ and flux $\pi \hbar / e$ are also constants, and this would assure the permanence of charge and flux. The corresponding conservation laws, including the premetric Maxwell equations (10), are thereby implied. The only problem with this argument is that flux occurs in quantized form only under special circumstances. Closely related ideas were put forward by Peres [20, 21].

Many electromagnetic theories fulfill, indeed, electric charge and magnetic flux conservation (4) and (7), respectively. Let us quote as examples nonlinear Born-Infeld electrodynamics [1], the quantum field theoretically motivated pseudo-classical Heisenberg-Euler electrodynamics [6], and, as a more recent case, the axion type electrodynamics of Carroll, Field, and Jackiw [2]. Itin [7] has shown that the CFJ-electrodynamics can be put 
in premetric form. We just have to assume the constitutive law $H={ }^{\star} F+a F$, with the metric-dependent Hodge star * and the scalar function $a:=v t / 2$, where $v$ is an absolute (i.e., prescribed) field and $t$ the time coordinate. A violation of the Lorentz symmetry is obtained by postulating this explicit (and noncovariant) time dependence of the constitutive tensor.

As we have seen, premetric electrodynamics turns out to be a useful framework for classifying different models of electrodynamics.

\section{LOCAL AND LINEAR SPACETIME RELATION}

The premetric framework, which we discussed so far, is incomplete. As we can read off from (10), we have $3+3=6$ equations for determining the time evolution of the electromagnetic field $(H ; F)$. However, the latter is described by the $6+6=12$ components $\left(\mathcal{H}_{a}, \mathcal{D}_{a b}=-\mathcal{D}_{b a} ; E_{a}, B_{a b}=-B_{b a}\right)$, with $a, b, \cdots=1,2,3$. Thus 6 equations are missing.

Guided by what we know from ordinary Maxwell-Lorentz electrodynamics, we will assume that the excitation $H$ is a functional of the field strength $F$. For vacuum spacetime, such a relation is expected to be local, i.e., no integrals are involved linking $H\left(x^{i}\right)$ to $F$ at another event (no heredity effects). Furthermore, linearity will be assumed (linear response). Accordingly, a local and linear operator $\kappa$ will be postulated to exist, that is, $H=\kappa(F)$. In components $H=H_{\alpha \beta} \vartheta^{\alpha} \wedge \vartheta^{b} / 2$ etc., this spacetime relation reads,

$$
H_{\alpha \beta}=\frac{1}{2} \kappa_{\alpha \beta}^{\gamma \delta} F_{\gamma \delta} .
$$

The constitutive tensor of spacetime $\kappa_{\alpha \beta}{ }^{\gamma \delta}$ can also be transformed into holonomic coordinates: $\kappa_{\alpha \beta}{ }^{\gamma \delta}=e_{\alpha}^{i} e_{\beta}^{j} e_{k}{ }^{\gamma} e_{l}^{\delta} \kappa_{i j}{ }^{k l}$ (see Schouten[28]). Sometimes it is more convenient to apply a constitutive tensor with all indices as superscripts. We raise two indices with the help of the Levi-Civita symbol and find

$$
\chi^{\alpha \beta \gamma \delta}:=\frac{1}{2} \epsilon^{\alpha \beta \mu \nu} \kappa_{\mu \nu}^{\gamma \delta} .
$$

Alternatively, we can introduce a 6 -dimensional vector space with the collective indices $I, K, \cdots=1, \ldots, 6 \equiv 01,02,03,23,31,12$. Then (12) can be rewritten as

$$
H_{I}=\kappa_{I}^{K} F_{K}=\hat{\epsilon}_{I M} \chi^{M K} F_{K} .
$$


All information on the electromagnetic properties of spacetime is encoded in the 36 components of $\kappa$ or $\chi$, respectively. It is straightforward to decompose $\chi$ irreducibly under the linear group $G L(4, R)$. The $6 \times 6$ matrix $\chi^{I K}$ decomposes in its symmetric tracefree, its antisymmetric, and its trace pieces:

$$
\begin{aligned}
& \chi^{I K}=\left(\chi^{(I K)}-\frac{1}{6} \epsilon^{I K} \chi_{L}{ }^{L}\right)+\chi^{[I K]}+\frac{1}{6} \epsilon^{I K} \chi_{L}{ }^{L}, \\
& 36=\quad \begin{array}{lllll}
20 & \oplus & 15 & \oplus & 1 .
\end{array}
\end{aligned}
$$

Translated into the 4D formalism, we find

$$
\chi^{i j k l}=\underbrace{(1) \chi^{i j k l}}_{20, \text { principal }}+\underbrace{\epsilon^{i j m[k} \not_{m}^{l]}-\epsilon^{k l m[i} \not_{m}^{j]}}_{15, \text { skewon }}+\underbrace{\epsilon^{i j k l} \alpha}_{1, \text { axion }} .
$$

We indicated the names and the number of independent components in the last equation. In conventional Maxwell-Lorentz electrodynamics, only the principal part ${ }^{(1)} \chi^{i j k l}$ is assumed to contribute to the spacetime relation. The tracefree $4 \times 4$ matrix $\not_{i}^{j}$ describes the skewon part and the axial (or pseudo) scalar $\alpha$ the axion part of $\chi$. Again, a transformation from anholonomic coordinates can be achieved by the usual rule $\chi^{i j k l}=e_{\alpha}^{i} e_{\beta}^{j} e_{\gamma}^{k} e_{\delta}^{l} \chi^{\alpha \beta \gamma \delta}$.

We substitute (16) in the holonomic version of (12). This yields the spacetime relation

$$
H_{i j}=\frac{1}{2}{ }^{(1)} \kappa_{i j}{ }^{k l} F_{k l}+2 \not_{[i}^{k} F_{j] k}+\alpha F_{i j}
$$

Still, the $6 \mathrm{D}$ version in $3+3$ form may be nearer to our intuition. It reads, see 4] for details $(a, b=1,2,3)$,

$$
\left(\begin{array}{l}
\mathcal{H}_{a} \\
\mathcal{D}^{a}
\end{array}\right)=\left(\begin{array}{cc}
\mathcal{C}^{b} & \mathcal{B}_{b a} \\
\mathcal{A}^{b a} & \mathcal{D}_{b}{ }^{a}
\end{array}\right)\left(\begin{array}{c}
-E_{b} \\
B^{b}
\end{array}\right)
$$

with the constitutive tensors

$$
\kappa_{I}{ }^{K}=\left(\begin{array}{cc}
\mathcal{C}^{b}{ }_{a} & \mathcal{B}_{b a} \\
\mathcal{A}^{b a} & \mathcal{D}_{b}{ }^{a}
\end{array}\right), \quad \chi^{I K}=\left(\begin{array}{cc}
\mathcal{B}_{a b} & \mathcal{D}_{a}{ }^{b} \\
\mathcal{C}^{a}{ }_{b} & \mathcal{A}^{a b}
\end{array}\right) .
$$

For material media, such constitutive tensors are well-known, see Lindell et al. [17] and Mackay and Lakhtakia [18], for example. Mackay and Lakhtakia consider a material with electric/magnetic cross-terms, i.e., $\mathcal{C}^{a}{ }_{b}$ and $\mathcal{D}_{a}{ }^{b}$ are nonvanishing. (However, they require, see also [13], the axion part to vanish.) It is convenient to write down explicitly the contributions 
of the principal, the skewon, and the axion parts to the constitutive 3-matrices, cf. (16):

$$
\begin{aligned}
\mathcal{A}^{a b} & =-\varepsilon^{a b}-\epsilon^{a b c} \not_{c}{ }^{0}, \\
\mathcal{B}_{a b} & =\mu_{a b}^{-1}+\hat{\epsilon}_{a b c} \not_{0}{ }^{c}, \\
\mathcal{C}^{a}{ }_{b} & =\gamma^{a}{ }_{b}-\left(\oiint_{b}{ }^{a}-\delta_{b}^{a} \not_{c}{ }^{c}\right)+\alpha \delta_{b}^{a}, \\
\mathcal{D}_{a}{ }^{b} & =\gamma^{b}{ }_{a}+\left(\oiint_{a}{ }^{b}-\delta_{a}^{b} \not_{c}{ }^{c}\right)+\alpha \delta_{a}^{b} .
\end{aligned}
$$

The set of the two symmetric matrices $\varepsilon^{a b}=\varepsilon^{b a}, \mu_{a b}^{-1}=\mu_{b a}^{-1}$ plus the traceless matrix $\gamma^{a}{ }_{b}$ (with $\gamma_{c}^{c}=0$ ) comprise the principal part of the constitutive tensor. Usually, $\varepsilon^{a b}$ is called permittivity tensor and $\mu_{a b}^{-1}$ reciprocal permeability tensor ("impermeability" tensor), since they describe the polarizability and magnetizability properties of a medium, respectively. The cross-term $\gamma_{b}^{a}$ is related to the Fresnel-Fizeau effects. The skewon contributions in (20) and (21) are responsible for the electric and magnetic Faraday effects, respectively, whereas skewon terms in (22) and (23) describe optical activity.

\section{PROPAGATION OF WAVES: FRESNEL-HADAMARD APPROACH}

Wave propagation is a very important physical phenomenon in classical field theory. In electrodynamics, one usually distinguishes between physical optics and geometric optics. We will confine our attention to the latter case. The appropriate formalism is then provided by the Fresnel-Hadamard approach in which a wave is described in terms of the propagation of a discontinuity of the field. Let us define the surface of discontinuity $S$ locally by a function $\Phi$ such that $\Phi=$ const on $S$. As usual, we denote by $[\mathcal{F}](x)$ the discontinuity of a function $\mathcal{F}$ across $S$, and $q:=d \Phi$ is the wave covector. Then for an ordinary wave, the geometric Hadamard conditions are satisfied across $S$ :

$$
\begin{aligned}
& {[H]=0, \quad[d H]=q \wedge h,} \\
& {[F]=0, \quad[d F]=q \wedge f .}
\end{aligned}
$$

The 2-forms $h$ and $f$ describe the jumps of the derivatives of the electromagnetic fields. Using Maxwell's equations (5) and (7), we find

$$
q \wedge h=0, \quad q \wedge f=0 .
$$

The latter equation can evidently be solved by $f=q \wedge a$, with an arbitrary covector $a$. Now we use the spacetime relation $H=\kappa(F)$. The corresponding relation for the jump 2-forms 
reads $h=\kappa(f)=\widetilde{\kappa}(f)+\alpha f$, where we separated the axion piece and denoted the rest of the constitutive relation (17) by the $\widetilde{\kappa}$. Substituting this into (26), we finally obtain

$$
q \wedge h=q \wedge \widetilde{\kappa}(q \wedge a)=0
$$

The last equation can be considered as a kind of Fourier transform of the tensor analytical formula $\partial_{\beta}\left(\widetilde{\chi}^{\alpha \beta \gamma \delta} \partial_{\gamma} A_{\delta}\right)=0$, see Post [22], Eq.(9.40).

\section{A. Generalized Fresnel equation and Tamm-Rubilar tensor}

Now we are in a position to derive the Fresnel equation for the wave covector $q$. As a preliminary step we recall that a 3 -form in $4 \mathrm{D}$ has four components. Consequently, we can conveniently recast the 3 -form equation (27) into an equivalent 4-form equation by multiplication with $\vartheta^{\alpha}=e_{i}{ }^{\alpha} d x^{i}$. Recalling that $a=a_{\beta} \vartheta^{\beta}$, we find

$$
q \wedge \vartheta^{\alpha} \wedge \widetilde{\kappa}\left(q \wedge \vartheta^{\beta}\right) a_{\beta}=q \wedge \vartheta^{\alpha} \wedge \frac{1}{2} \widetilde{\kappa}_{\mu \nu}{ }^{\gamma \beta} q_{\gamma} a_{\beta} \vartheta^{\mu} \wedge \vartheta^{\nu}=0
$$

This algebraic system for the covector $a$ obviously admits the gauge freedom $a_{\beta} \rightarrow a_{\beta}+q_{\beta} \varphi$ with an arbitrary function $\varphi$. In order to deal with this problem, we choose the first leg of the local anholonomic coframe as $\vartheta^{\hat{0}} \stackrel{*}{=} q$. This can always be done without restricting the generality of our consideration. Then (28) reduces to

$$
\frac{1}{2} \widetilde{\kappa}_{\mu \nu}^{\hat{0} \beta} a_{\beta} \vartheta^{\hat{0}} \wedge \vartheta^{\alpha} \wedge \vartheta^{\mu} \wedge \vartheta^{\nu} \stackrel{*}{=} 0 \Rightarrow \frac{1}{2} \epsilon^{\hat{0} a c d} \widetilde{\kappa}_{c d}^{\hat{0} b} a_{b} \stackrel{*}{=} 0
$$

or, equivalently, to the system of three algebraic equations for the three unknown components $a_{b}$ :

$$
W^{a b} a_{b} \stackrel{*}{=} \hat{0} \quad \text { with } \quad W^{a b}:=\widetilde{\chi}^{\hat{0} a \hat{0} b} .
$$

Note that the gauge-dependent $a_{\hat{0}}$ eventually disappeared. A nontrivial solution of the system obtained exists if and only if the corresponding determinant vanishes,

$$
\mathcal{W}:=\operatorname{det} W^{a b} \stackrel{*}{=} \frac{1}{3 !} \hat{\epsilon}_{a b c} \hat{\epsilon}_{d e f} W^{a d} W^{b e} W^{c f} \stackrel{*}{=} 0
$$

or, substituting the components of the $3 \times 3$ matrix explicitly,

$$
\mathcal{W} \stackrel{*}{=} \frac{1}{3 !} \hat{\epsilon}_{a b c} \hat{\epsilon}_{d e f} \widetilde{\chi}^{\hat{0} a \hat{0} d} \widetilde{\chi}^{\hat{0} b \hat{e} e} \widetilde{\chi}^{\hat{0} c \hat{0} f} \stackrel{*}{=} 0 .
$$


Quite remarkably, we can rewrite the last equation as a 4D-covariant equation. Indeed, because of $\hat{\epsilon}_{a b c} \equiv \hat{\epsilon}_{\hat{0} a b c}$, after some algebra, we find

$$
\mathcal{W}=\frac{\theta^{2}}{4 !} \hat{\epsilon}_{m n p q} \hat{\epsilon}_{r s t u} \widetilde{\chi}^{m n r i} \widetilde{\chi}^{j p s k} \widetilde{\chi}^{l q t u} q_{i} q_{j} q_{k} q_{l}
$$

Here $\theta:=\operatorname{det}\left(e_{i}{ }^{\alpha}\right)$. Since, by assumption, the 1 -forms $\vartheta^{\alpha}$ constitute a basis of the cotangent space, $\theta$ is always nonvanishing.

Let us now define 4th-order Tamm-Rubilar tensor density of weight +1 ,

$$
\mathcal{G}^{i j k l}(\chi):=\frac{1}{4 !} \hat{\epsilon}_{m n p q} \hat{\epsilon}_{r s t u} \chi^{m n r(i} \chi^{j|p s| k} \chi^{l) q t u}
$$

In $n$ dimensions a totally symmetric tensor of rank $p$ has $\left(\begin{array}{c}n+p-1 \\ p\end{array}\right)=\left(\begin{array}{c}n-1+p \\ n-1\end{array}\right)$ independent components. Accordingly, in four dimensions the TR-tensor (34) has 35 independent components. Because $\chi^{i j k l}=\tilde{\chi}^{i j k l}+\alpha \epsilon^{i j k l}$, the total antisymmetry of the Levi-Civita $\epsilon$ evidently yields $\mathcal{G}(\chi)=\mathcal{G}(\tilde{\chi})$. Thus, starting from (33) and discarding the irrelevant nonvanishing factor $\theta^{2}$, we arrive at the generally covariant 4-dimensional (extended) Fresnel equation

$$
\mathcal{G}^{i j k l}(\chi) q_{i} q_{j} q_{k} q_{l}=0
$$

This quartic equation in the components $q_{i}$ of the wave covector $q$ was derived from a determinant of a $3 \times 3$ matrix. Apparently, the wave covectors $q$ lie on a quartic Fresnel wave surface in general, which, incidentally, is not exactly what we are observing in vacuum at the present epoch of our universe.

\section{B. Decomposing Fresnel equation into time and space pieces}

Physically, the zeroth component component of the wave covector is interpreted as the frequency of a "photon", whereas the spatial 3-vector part represents its momentum. Accordingly, the physical contents of the Fresnel equation becomes more transparent after we carefully separate it into its time and its space pieces. It is convenient to denote the 
independent components of the TR-tensor (34) as follows:

$$
\begin{gathered}
M:=\mathcal{G}^{0000}=\operatorname{det} \mathcal{A} \\
M^{a}:=4 \mathcal{G}^{000 a}=-\hat{\epsilon}_{b c d}\left(\mathcal{A}^{b a} \mathcal{A}^{c e} \mathcal{C}^{d}{ }_{e}+\mathcal{A}^{a b} \mathcal{A}^{e c} \mathcal{D}_{e}{ }^{d}\right) \\
M^{a b}:=6 \mathcal{G}^{00 a b}=\frac{1}{2} \mathcal{A}^{(a b)}\left[\left(\mathcal{C}^{d}{ }_{d}\right)^{2}+\left(\mathcal{D}_{c}{ }^{c}\right)^{2}-\left(\mathcal{C}^{c}{ }_{d}+\mathcal{D}_{d}{ }^{c}\right)\left(\mathcal{C}^{d}{ }_{c}+\mathcal{D}_{c}{ }^{d}\right)\right] \\
+\left(\mathcal{C}^{d}{ }_{c}+\mathcal{D}_{c}{ }^{d}\right)\left(\mathcal{A}^{c(a} \mathcal{C}^{b)}{ }_{d}+\mathcal{D}_{d}{ }^{(a} \mathcal{A}^{b) c}\right)-\mathcal{C}^{d}{ }_{d} \mathcal{A}^{c(a} \mathcal{C}^{b)}{ }_{c} \\
-\mathcal{D}_{c}{ }^{(a} \mathcal{A}^{b) c} \mathcal{D}_{d}{ }^{d}-\mathcal{A}^{d c} \mathcal{C}^{(a}{ }_{c} \mathcal{D}_{d}{ }^{b)}+\left(\mathcal{A}^{(a b)} \mathcal{A}^{d c}-\mathcal{A}^{d(a} \mathcal{A}^{b) c}\right) \mathcal{B}_{d c}, \\
M^{a b c}:=4 \mathcal{G}^{0 a b c}=\epsilon^{d e(c \mid}\left[\mathcal{B}_{d f}\left(\mathcal{A}^{a b)} \mathcal{D}_{e}{ }^{f}-\mathcal{D}_{e}{ }^{a} \mathcal{A}^{b) f}\right)\right. \\
\left.+\mathcal{B}_{f d}\left(\mathcal{A}^{a b)} \mathcal{C}^{f}{ }_{e}-\mathcal{A}^{f \mid a} \mathcal{C}^{b)}\right)+\mathcal{C}^{a}{ }_{f} \mathcal{D}_{e}{ }^{b)} \mathcal{D}_{d}{ }^{f}+\mathcal{D}_{f}{ }^{a} \mathcal{C}^{b)} \mathcal{C}^{f}{ }_{d}\right] \\
M^{a b c d}:=\mathcal{G}^{a b c d}=\epsilon^{e f(c} \epsilon^{|g h| d} \mathcal{B}_{h f}\left[\frac{1}{2} \mathcal{A}^{a b)} \mathcal{B}_{g e}-\mathcal{C}^{a}{ }_{e} \mathcal{D}_{g}{ }^{b)}\right]
\end{gathered}
$$

Then the Fresnel equation (35) in decomposed form reads

$$
q_{0}^{4} M+q_{0}^{3} q_{a} M^{a}+q_{0}^{2} q_{a} q_{b} M^{a b}+q_{0} q_{a} q_{b} q_{c} M^{a b c}+q_{a} q_{b} q_{c} q_{d} M^{a b c d}=0 .
$$

\section{REDUCING THE QUARTIC WAVE SURFACE TO THE LIGHT CONE}

\section{A. Maxwell-Lorentz electrodynamics in vacuum}

As a first example, let us demonstrate that our formalism yields the correct result for the conventional Maxwell-Lorentz spacetime relation with the constitutive tensor density

$$
\chi^{i j k l}=2 \lambda_{0} \sqrt{-g} g^{i[k \mid} g^{j \mid l]}=\lambda_{0} \sqrt{-g}\left(g^{i k} g^{j l}-g^{i l} g^{j k}\right) .
$$

Here $\lambda_{0}=\sqrt{\varepsilon_{0} / \mu_{0}}$ is the so-called vacuum impedance. A spacetime metric $g_{i j}$ is assumed on the manifold. Substituting (42) into (34), we can calculate the corresponding TR-tensor density straightforwardly:

$$
\mathcal{G}^{i j k l}=-\lambda_{0}^{3} \sqrt{-g} g^{(i j} g^{k l)}=\frac{\lambda_{0}^{3}}{3} \sqrt{-g}\left(g^{i j} g^{k l}+g^{k j} g^{i l}+g^{l j} g^{i k}\right) .
$$

As a result, the quartic wave surface (35) reduces to the usual light cone (twice):

$$
\mathcal{G}^{i j k l} q_{i} q_{j} q_{k} q_{l}=-\lambda_{0}^{3} \sqrt{-g}\left(g^{i j} q_{i} q_{j}\right)\left(g^{k l} q_{k} q_{l}\right)=0
$$




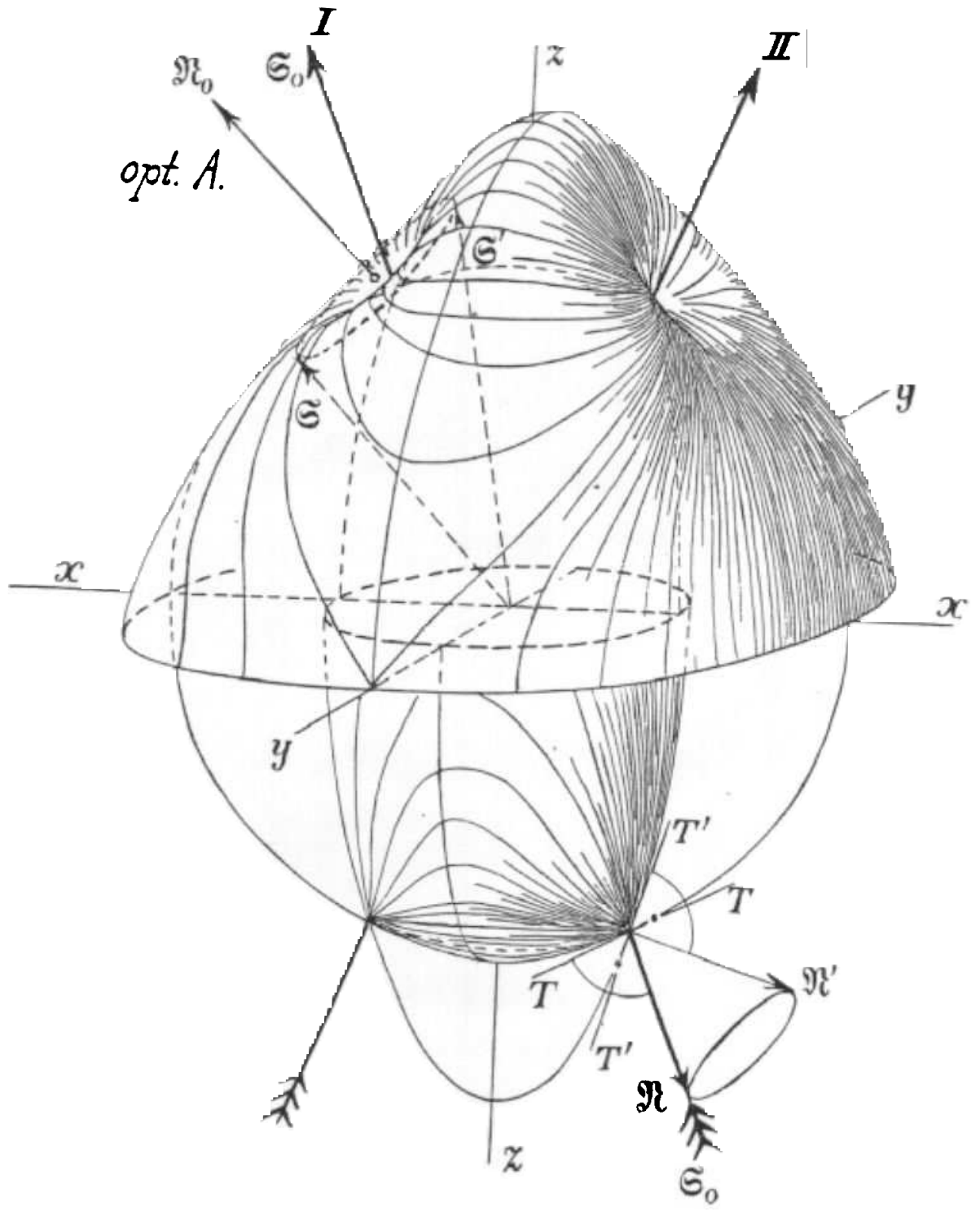

FIG. 2: Clemens Schaefer's drawing 25] of a quartic ray surface for $-\mathcal{A} \equiv \varepsilon=\varepsilon^{T}, \mathcal{B} \equiv \mu^{-1}=1$, and $\mathcal{C}=0, \mathcal{D}=0$ : One ray vector has 4 in general different cuts with the quartic surface. The Fresnel equation (48), which describes the case under consideration, defines a quartic wave surface that is dual to the ray surface of this figure. Accordingly, both surfaces encompass the same information and look, in fact, fairly similar. Corresponding details are discussed in the Appendix.

\section{B. Constitutive tensor with vanishing electric/magnetic cross-terms}

The matrices $\mathcal{D}_{b}{ }^{a}$ and $\mathcal{C}^{b}{ }_{a}$ in the general spacetime relation (18) describe the mixing of electric and magnetic fields. Let us consider the case when these cross-terms are absent, $\mathcal{C}^{b}{ }_{a}=0, \mathcal{D}_{b}{ }^{a}=0$, whereas $\mathcal{A}^{a b}$ and $\mathcal{B}_{a b}$ are left asymmetric. Physically, they are interpreted 
as the generalized permittivity and permeability tensors, respectively. Now the $M$-tensor densities (36)-(40) simplify appreciably: $M^{a}=0$ and $M^{a b c}=0$, while

$$
\begin{aligned}
M & =\operatorname{det} \mathcal{A} \\
M^{a b} & =\left(\mathcal{A}^{(a b)} \mathcal{A}^{d c}-\mathcal{A}^{d(a} \mathcal{A}^{b) c}\right) \mathcal{B}_{d c}, \\
M^{a b c d} & =\frac{1}{2} \epsilon^{e f(a} \epsilon^{|g h| b} \mathcal{A}^{c d)} \mathcal{B}_{g e} \mathcal{B}_{h f} .
\end{aligned}
$$

The time and space decomposed Fresnel equation (41) then reduces to

$$
M q_{0}^{4}+M^{a b} q_{a} q_{b} q_{0}^{2}+M^{a b c d} q_{a} q_{b} q_{c} q_{d}=0 .
$$

This bi-quadratic equation can be solved for the frequency square and yields a Finsler metric, see Rubilar [23],

$$
\left(q_{0}^{2}+\frac{M^{a b} q_{a} q_{b}+\sqrt{\Delta}}{2 M}\right)\left(q_{0}^{2}+\frac{M^{a b} q_{a} q_{b}-\sqrt{\Delta}}{2 M}\right)=0
$$

with $\Delta:=\left(M^{a b} q_{a} q_{b}\right)^{2}-4 M M^{a b c d} q_{a} q_{b} q_{c} q_{d}$. A unique light cone can be recovered, provided $\Delta=0$ or

$$
4 M M^{a b c d}=M^{(a b} M^{c d)}
$$

If this sufficient condition for the existence of a light cone is fulfilled, then the quartic Fresnel wave surface reduces to

$$
\left(q_{0}^{2}+\frac{M^{a b} q_{a} q_{b}}{2 M}\right)^{2}=0 \quad \text { or } \quad\left(g_{\text {opt }}^{i k} q_{i} q_{k}\right)^{2}=0 .
$$

Here the optical metric is introduced with the components $g_{\mathrm{opt}}^{00}=2 M, g_{\mathrm{opt}}^{a b}=M^{a b}$.

If the algebraic condition (50) is not satisfied, the form of the resulting quartic wave surface is extremely complicated. For example, even in case when $\mathcal{B}_{a b} \sim \delta_{a b}$ and $\mathcal{A}^{a b}=\mathcal{A}^{b a}$ (which describes also the light propagation in an anisotropic purely dielectric crystal), the quartic Fresnel surface still looks highly nontrivial, see Fig. 2.

\section{Sufficient condition for unique light cone: electric/magnetic reciprocity}

The algebraic condition (50) imposes a highly nontrivial constraint on the components of the constitutive matrices. Although a particular solution (for the homogeneous dielectric/magnetic medium) can be easily derived, the general solution of (150) is unknown. There 
exists, however, another sufficient condition for the reduction of the quartic Fresnel surface to a unique light cone which admits a complete solution. Let us put skewon and axion fields to zero $\not_{i}{ }^{j}=0, \alpha=0$. Then the spacetime relation (17) contains only the first term on the right-hand-side with (18) constructed of the symmetric matrices $\mathcal{A}^{a b}=\mathcal{A}^{b a}, \mathcal{B}_{a b}=\mathcal{B}_{b a}$ and the traceless matrices $\mathcal{D}_{a}{ }^{b}=\mathcal{C}^{b}{ }_{a}$, with $\mathcal{C}^{c}{ }_{c}=0$.

Since the times of Maxwell and Heaviside, in the equations of electrodynamics a certain symmetry was noticed between the electric and the magnetic quantities and was used in theoretical discussions. We formulate electric/magnetic reciprocity as follows [4]: the energymomentum current (11) is electric/magnetic reciprocal, i.e., it remains invariant $\Sigma_{\alpha} \rightarrow \Sigma_{\alpha}$ under the transformation

$$
H \rightarrow \zeta F, \quad F \rightarrow-\frac{1}{\zeta} H,
$$

with the twisted zero-form (pseudo-scalar function) $\zeta=\zeta(x)$ of dimension $[\zeta]=[H] /[F]=$ $q^{2} / h=1 /$ resistance.

We now require the spacetime relation also to be electric/magnetic reciprocal. Then

$$
\zeta F_{I}={ }^{(1)} \kappa_{I}{ }^{K}\left(-\frac{1}{\zeta} H_{K}\right) \quad \text { or } \quad-\zeta^{2} F_{I}={ }^{(1)} \kappa_{I}{ }^{K(1)} \kappa_{K}{ }^{L} F_{L}
$$

Consequently, the constitutive tensor satisfies a closure relation:

$$
{ }^{(1)} \kappa_{I}{ }^{K(1)} \kappa_{K}{ }^{L}=-\zeta^{2} \delta_{I}^{L}
$$

Therefrom we find $\zeta^{2}=-\frac{1}{6} \operatorname{Tr}\left({ }^{(1)} \kappa^{2}\right)=: \lambda^{2}$, and hence $\left({ }^{(1)} \kappa\right)^{2}=-\lambda^{2} \mathbf{1}_{6}$. Thus, symbolically, we can write ${ }^{(1)} \kappa=\lambda \sqrt{-\mathbf{1}_{6}}$. With $J:=\sqrt{-\mathbf{1}_{6}}$, we can then define the duality operator

$$
\#:=J=\frac{1}{\lambda}{ }^{(1)} \kappa \text {. }
$$

Besides the closure property $J^{2}=-\mathbf{1}_{6}$, this operator is also symmetric by construction, $J(\phi) \wedge \psi=\phi \wedge J(\psi)$, for all 2-forms $\phi$ and $\psi$. As a result, the spacetime relation reads:

$$
H=\lambda^{\#} F \quad \text { with } \quad[\lambda]=1 / \text { resistance } .
$$

Using the $3 \times 3$-matrix parametrization (18) of the constitutive tensor, we can solve the closure relation explicitly. In matrix notation, $J=\left(\begin{array}{cc}\mathcal{C} & \mathcal{B} \\ \mathcal{A} & \mathcal{C}^{T}\end{array}\right)$. Thus, the closure relation $J^{2}=-1$ reads:

$$
\mathcal{C}^{2}+\mathcal{A B}=-1, \quad \mathcal{B C}+\mathcal{C}^{T} \mathcal{B}=0, \quad \mathcal{C} \mathcal{A}+\mathcal{A C}^{T}=0
$$


The solution is obtained straightforwardly in terms of $\mathcal{A}$ and an arbitrary skew-symmetric matrix $\widehat{K}=-\widehat{K}^{T}$ :

$$
\mathcal{B}=-\mathcal{A}^{-1}\left[1+(\widehat{K} \mathcal{A})^{2}\right], \quad \mathcal{C}=\widehat{K} \mathcal{A}^{-1}
$$

Now we can substitute this solution into (36)-(40) and find the TR tensor density:

$$
\begin{aligned}
M & =\operatorname{det} \mathcal{A}, \\
M^{a} & =4 \widehat{k}^{a} \\
M^{a b} & =-2 \mathcal{A}^{a b}+6 \widehat{k}^{a} \widehat{k}^{b} / \operatorname{det} \mathcal{A}, \\
M^{a b c} & =4\left(-\mathcal{A}^{(a b} \widehat{k}^{c)}+\widehat{k}^{a} \widehat{k}^{b} \widehat{k}^{c} / \operatorname{det} \mathcal{A}\right) / \operatorname{det} \mathcal{A}, \\
M^{a b c d} & =\left(\mathcal{A}^{(a b} \mathcal{A}^{c d)}-2 \mathcal{A}^{(a b} \widehat{k}^{c} \widehat{k}^{d)} / \operatorname{det} \mathcal{A}+\widehat{k}^{a} \widehat{k}^{b} \widehat{k}^{c} \widehat{k}^{d} / \operatorname{det} \mathcal{A}\right) / \operatorname{det} \mathcal{A} .
\end{aligned}
$$

Here we denote $\widehat{k}^{a}:=\mathcal{A}^{a b} \epsilon_{b c d} \widehat{K}^{c d}$. Finally, substituting this into (41), we can verify that the Fresnel equation reduces to

$$
\left(g^{i k} q_{i} q_{k}\right)^{2}=0
$$

Here the spacetime metric is constructed from the components of the constitutive matrices as follows

$$
g^{i k}=\frac{1}{\sqrt{-\operatorname{det} \mathcal{A}}}\left(\begin{array}{cc}
\operatorname{det} \mathcal{A} & \widehat{k}^{b} \\
\widehat{k}^{a} & -\mathcal{A}^{a b}+\widehat{k}^{a} \widehat{k}^{b} / \operatorname{det} \mathcal{A}
\end{array}\right) .
$$

It is not difficult to prove that this metric has Lorentzian signature for every matrix $\mathcal{A}^{a b}$.

\section{DISCUSSION AND CONCLUSION}

The main goal of this paper was to demonstrate that the light cone can be recovered from a local and linear spacetime relation of classical electrodynamics. Closure and symmetry of this spacetime relation are sufficient conditions that guarantee the reduction of the general quartic Fresnel wave surface to a unique light cone. The closure relation alone, without the assumption of symmetry, is not sufficient for the recovery of the light cone structure 24].

As a result of the reduction of the quartic Fresnel surface, we find the spacetime metric with Minkowski (aka Lorentz) signature. This is intimately related to the minus sign in the reciprocity transformation (52) and the closure relation (54). A plus sign would yield the wrong Euclidean signature. Our approach shows that one can treat the duality operator \# as a metricfree predecessor of the Hodge operator $\star$ that appears in 
the standard Maxwell-Lorentz spacetime relation: \# (duality operator) of Eq. (55) $\longrightarrow$ * (Hodge operator) of Eq. (2).

Summarizing, the conformal part of the metric, that is, the light cone, naturally emerges in our framework from a local and linear spacetime relation. In this sense, the light cone (and the spacetime metric) is an electromagnetic construct.

\section{Acknowledgments}

Different versions of this paper have been given as seminars in Vienna, Columbia/Missouri, Jena, and Bath. Respective discussions with W. Kummer, B. Mashhoon, G. Neugebauer \& A. Wipf, and with D. Vassiliev are gratefully acknowledged. We also thank Yakov Itin and Claus Lämmerzahl for most helpful remarks. Our work has been supported by the DFG project HE 528/20-1.

\section{APPENDIX: WAVE AND RAY SURFACES}

The drawing in Fig. 2 doesn't depict the wave surface but rather the so-called ray surface which is dual to the wave surface. In the particular case of Fig. 2, the propagation in an anisotropic dielectric medium is discussed with $\mathcal{A}^{a b}=-\frac{1}{c} \varepsilon^{a b}, \varepsilon^{a b}=\operatorname{diag}\left(\varepsilon_{1}, \varepsilon_{2}, \varepsilon_{3}\right)$, and $\mathcal{B}_{a b}=c \delta_{a b}$ (with $c$ as speed of light). Then we can immediately verify from (45)-(47) that $M=-\varepsilon_{1} \varepsilon_{2} \varepsilon_{3} / c^{3}, M^{a b c d}=c \delta^{(a b} \varepsilon^{c d)}$, and

$$
M^{a b}=\frac{1}{c}\left(\begin{array}{ccc}
\varepsilon_{1}\left(\varepsilon_{2}+\varepsilon_{3}\right) & 0 & 0 \\
0 & \varepsilon_{2}\left(\varepsilon_{1}+\varepsilon_{3}\right) & 0 \\
0 & 0 & \varepsilon_{3}\left(\varepsilon_{1}+\varepsilon_{2}\right)
\end{array}\right) .
$$

As a result, the Fresnel equation for the wave surface (48) can be recast into the simple form

$$
\frac{\varepsilon_{1} q_{1}^{2}}{c^{2} \vec{q}^{2}-q_{0}^{2} \varepsilon_{1}}+\frac{\varepsilon_{2} q_{2}^{2}}{c^{2} \vec{q}^{2}-q_{0}^{2} \varepsilon_{2}}+\frac{\varepsilon_{3} q_{3}^{2}}{c^{2} \vec{q}^{2}-q_{0}^{2} \varepsilon_{3}}=0 .
$$

Here $\vec{q}^{2}=q_{1}^{2}+q_{2}^{2}+q_{3}^{2}$. In crystal optics one usually introduces a ray 4 -vector $s$ which is dual to the wave covector $q$, i.e., $s\rfloor q=0$, see Kiehn et al. [10]. The physical meaning of the ray vector is as follows: Its spatial part coincides with the Poynting vector $\vec{s}=\vec{E} \times \vec{H}$, whereas its time component describes the ray velocity. Then from (67) one can straightforwardly 
derive the dual equation of the Fresnel ray surface for the components of $s=\left\{s^{0}, s^{1}, s^{2}, s^{3}\right\}$ :

$$
\frac{\left(s^{1}\right)^{2}}{c^{2} \vec{s}^{2}-\left(s^{0}\right)^{2} \varepsilon_{1}}+\frac{\left(s^{2}\right)^{2}}{c^{2} \vec{s}^{2}-\left(s^{0}\right)^{2} \varepsilon_{2}}+\frac{\left(s^{3}\right)^{2}}{c^{2} \vec{s}^{2}-\left(s^{0}\right)^{2} \varepsilon_{3}}=0 .
$$

As we can see, the quartic surfaces (67) and (68) look pretty much similar, and thus depicting one of them in fact gives a good idea about the dual surface. In Fig. 2, we see the ray surface with $x=s^{0} s^{1} / \vec{s}^{2}, y=s^{0} s^{2} / \vec{s}^{2}, z=s^{0} s^{3} / \vec{s}^{2}$. The vectors $\mathfrak{S}_{0}, \mathfrak{S}$, and $\mathfrak{S}^{\prime}$ represent the particular rays related to the corresponding Poynting vectors, whereas $\mathfrak{N}_{0}, \mathfrak{N}$, and $\mathfrak{N}^{\prime}$ represent the wave fronts (propagating along the corresponding wave covectors $q$ ) dual to them. The Poynting vector $\vec{s}=\vec{E} \times \vec{H}$ describes the energy flux density, the wave vector $\vec{q} \sim \vec{D} \times \vec{B}$ the momentum density.

[1] M. Born and L. Infeld, Foundations of the new field theory, Proc. Roy. Soc. (London) A144 (1934) 425-451.

[2] S.M. Carroll, G.B. Field, R. Jackiw, Limits on a Lorentz and parity violating modification of electrodynamics, Phys. Rev. D41 (1990) 1231-1240.

[3] A. Einstein, Eine neue formale Deutung der Maxwellschen Feldgleichungen der Elektrodynamik (A new formal interpretation of Maxwell's field equations of electrodynamics), Sitzungsber. Königl. Preuss. Akad. Wiss. Berlin (1916) pp. 184-188; see also The collected papers of Albert Einstein. Vol.6, A.J. Kox et al., eds. (1996) pp. 263-269.

[4] F.W. Hehl and Yu.N. Obukhov, Foundations of Classical Electrodynamics: Charge, Flux, and Metric. Birkhäuser, Boston, MA (2003).

[5] F.W. Hehl and Yu.N. Obukhov, Electric/magnetic reciprocity in premetric electrodynamics with and without magnetic charge, and the complex electromagnetic field, Phys. Lett. A323 (2004) 169-175.

[6] W. Heisenberg and H. Euler, Folgerungen aus der Diracschen Theorie des Positrons (Consequences of Dirac's theory of the positron), Z. Phys. 98 (1936) 714-732.

[7] Y. Itin, Caroll-Field-Jackiw electrodynamics in the pre-metric framework, arXiv.org: hep-th/0403023 (6 pages) (2004).

[8] Y. Itin and F.W. Hehl, Is the Lorentz signature of the metric of spacetime electromagnetic in origin? Annals of Physics (NY), in print (2004); arXiv.org: gr-qc/0401016 
[9] G. Kaiser, Energy-momentum conservation in pre-metric electrodynamics with magnetic charges, arXiv.org: math-ph/0401028 (5 pages) (2004).

[10] R.M. Kiehn, G.P. Kiehn, and J.B. Roberds, Parity and time-reversal symmetry breaking, singular solutions, and Fresnel surfaces, Phys. Rev. A43 (1991) 5665-5671.

[11] F. Kottler, Maxwell'sche Gleichungen und Metrik (Maxwell's equations and metric), Sitzungsber. Akad. Wien IIa, 131 (1922) 119-146.

[12] A. Kovetz, Electromagnetic Theory, Oxford University Press, Oxford (2000).

[13] A. Lakhtakia, On the genesis of Post constraint in modern electromagnetism, arXiv.org: physics/0403042 (15 pages) (2004).

[14] C. Lämmerzahl and M.P. Haugan, On the interpretation of Michelson-Morley experiments, Phys. Lett. A282 (2001) 223-229.

[15] C. Lämmerzahl et al., OPTIS - An Einstein mission for improved tests of special and general relativity, Gen. Relativ. Grav. 36 (2004), to appear (31 pages).

[16] C. Lämmerzahl, A. Macias, H. Müller, Charge non-conservation: a general theoretical frame, in preparation $(2004 / 05)$.

[17] I.V. Lindell, A.H. Sihvola, S.A. Tretyakov, A.J. Viitanen, Electromagnetic waves in chiral and bi-Isotropic media, Artech House, Boston (1994).

[18] T.G. Mackay, A. Lakhtakia, Plane waves with negative phase velocity in Faraday chiral mediums, Phys. Rev. E69 (2004) article 026602 (9 pages).

[19] A. Peres, Electromagnetism, geometry, and the equivalence principle, Ann. Phys. (NY) 19 (1962) 279-286.

[20] A. Peres, The speed of light need not be constant, arXiv.org: gr-qc/0210066 (3 pages) (2002).

[21] A. Peres, Variability of fundamental constants, Int. J. Mod. Phys. D12 (2003) 1751-1754.

[22] E.J. Post, Formal Structure of Electromagnetics - General Covariance and Electromagnetics. North Holland, Amsterdam (1962) and Dover, Mineola, NY (1997).

[23] G.F. Rubilar, Linear pre-metric electrodynamics and deduction of the lightcone, Thesis (University of Cologne, June 2002); see Ann. Phys. (Leipzig) 11 (2002) 717-782.

[24] G.F. Rubilar, Yu.N. Obukhov, and F.W. Hehl, Generally covariant Fresnel equation and the emergence of the light cone structure in linear pre-metric electrodynamics, Int. J. Mod. Phys. D11 (2002) 1227-1242.

[25] C. Schaefer, Einführung in die theoretische Physik: Dritter Band, erster Teil — Elektrody- 
namik und Optik, de Gruyter, Berlin (1932).

[26] F. Scheck, Theoretische Physik 3. Klassische Feldtheorie. Von der Elektrodynamik zu den Eichtheorien, Springer, Berlin (2004).

[27] M. Schönberg, Electromagnetism and gravitation, Rivista Brasileira de Fisica 1 (1971) 91-122.

[28] J.A. Schouten, Tensor Analysis for Physicists, 2nd ed. reprinted, Dover, Mineola, New York (1989).

[29] A. Sommerfeld, Elektrodynamik. Vorlesungen über Theoretische Physik, Band 3, Dieterich'sche Verlagsbuchhandlung, Wiesbaden (1948). English translation: A. Sommerfeld, Electrodynamics, Lectures in Theoretical Physics, vol. 3, Academic Press, New York (1952).

[30] R.A. Toupin, Elasticity and electro-magnetics, in: Non-Linear Continuum Theories, C.I.M.E. Conference, Bressanone, Italy 1965. C. Truesdell and G. Grioli, coordinators. Pp.203-342.

[31] C. Truesdell and R.A. Toupin, The Classical Field Theories, in Handbuch der Physik III/1, S. Flügge, ed. Springer, Berlin (1960) pp. 226-793. 